\title{
Effect of Powder Morphologies on the Property of Conductive Silicone Rubber Filled with Carbonyl Nickel Powder
}

\author{
WEIMIN XIAO, ${ }^{1,3}$ YONGPING LEI, ${ }^{1}$ ZHIDONG XIA,${ }^{1}$ XIN CHEN,${ }^{2}$ \\ YU HAN, ${ }^{2}$ JINGKAI NIE, ${ }^{2}$ and PEI HUANG ${ }^{1}$
}

1.-School of Material Science and Engineering, Beijing University of Technology, 100 Ping Le Yuan, Chaoyang District, Beijing 100022, People's Republic of China. 2.-Global Energy Interconnection Research Institute, Future City for Science and Technology, Changping District, Beijing 102209, People’s Republic of China. 3.—e-mail: weimin@emails.bjut.edu.cn

Conductive silicone rubbers filled with the spherical, flaky, and chain-spherical carbonyl nickel powder were prepared. The effects of powder morphologies on their electromagnetic and mechanical properties were analyzed. The electromagnetic shielding effectiveness (SE) and tensile strength of the rubbers varies with their powder morphologies: the $\mathrm{SE}$ values increase from the spherical and the chain-spherical to the flaky morphology in the frequency range of $100-400 \mathrm{MHz}$. In the range of $500-1500 \mathrm{MHz}$, the SE rises from the spherical and the flaky to the chain-spherical morphologies. In addition, the tensile strength increases from the spherical and the flaky to the chainspherical morphologies. These variations are related to the differences in the conductive network structure and the powder distribution in the rubber.

Key words: Carbonyl nickel powder, powder morphology, conductive silicone rubber, electromagnetic shielding property, mechanical property

\section{INTRODUCTION}

As a novel conductive and electromagnetic interference shielding material, conductive silicone rubbers play an important role in the electrical connection and electromagnetic protection of sensitive electronic components. To improve the conductive and electromagnetic performance, a number of materials have been researched in recent years, such as conductive silicone rubbers filled with nickel-coated carbon fibers, nickel-coated graphite, and silver-coated cenospheres. ${ }^{1-3}$

It is well known that the fillers have a significant influence on the application of the rubbers. Common fillers include metals (e.g., copper powder), carbon materials (e.g., carbon fibers) and core-shell particles (e.g., silver-coated cenosphere). They possess relatively high conductivity and are beneficial to the enhancement of the electromagnetic wave reflection of the rubbers. ${ }^{3-7}$ Another fillers include the

(Received February 12, 2017; accepted May 17, 2017;

published online June 7, 2017) particles of dielectric or magnetic dipoles (e.g., Co/ $\mathrm{SiO}_{2}$ nanosphere core-shell composites and carbonyl iron/nickel powder). They can absorb and attenuate the incident electromagnetic waves, then crunch the electromagnetic energy into heat energy, or weaken the electromagnetic waves' interference. $^{8-10}$

Carbonyl iron powders and carbonyl nickel powders have been applied in the electronic industry as magnetic fillers due to their high saturation magnetization, superior stability, and enhanced dispersion. ${ }^{11-14}$ Recently, it has been proved that the different morphology of carbonyl iron powder can influence the electromagnetic properties of the polymer filled with the powder., 95

However, little work has been carried out to understand the effects of the powder morphology on the electromagnetic wave-shielding properties of the conductive silicone rubbers filled with the carbonyl nickel powder. Although the carbonyl nickel and carbonyl iron powders possess good magnetic performances, the microstructures and properties of the two powders are different. ${ }^{14,15}$ Considering the excellent magnetic property and 
well-defined structure of the carbonyl nickel powder, the optimized morphology of the carbonyl nickel powder may provide another good choice for the electromagnetic property improvement of the rubbers. Therefore, the investigation of the conductive silicone rubbers filled with carbonyl nickel powder which has different powder morphology is necessary for excellent electromagnetic wave-shielding capabilities.

Primary work has been performed on the effect of powder morphology on the electromagnetic properties of materials. Wan ${ }^{16}$ found that the varying morphologies of the FeCo-coated carbon fibers improved the electromagnetic properties of the polymer with the fibers at microwave frequency. Jazirehpour ${ }^{17}$ indicated that the magnetite nanostructures with different morphologies (tube, urchin and dendrite) have a major role in the microwave magnetic and dielectric properties of the polymers with the nanostructures.

In this paper, carbonyl nickel powders with three powder morphologies have been selected because of their good electromagnetic properties in previous works. The effects of the powder morphologies on the electromagnetic wave-shielding properties of the conductive silicone rubbers filled with the powders are discussed so as to understand the relationship between the properties and the powder morphologies.

\section{EXPERIMENTAL}

\section{Materials and Preparation}

Spherical, flaky and chain-spherical carbonyl nickel powders (99.5 wt.\%) were selected as three kinds of experimental powders because of their good electromagnetic properties. The average particle size of the powders was about $6 \mu \mathrm{m}, 6 \mu \mathrm{m}$, and $1.5 \mu \mathrm{m}$, respectively. The flaky carbonyl nickel powder was obtained by putting the spherical carbonyl nickel powder through a milling process.

Conductive silicone rubber filled with the carbonyl nickel powder was prepared by mixing the pure silicone rubber, vulcanizing agent and carbonyl nickel powder at $15^{\circ} \mathrm{C}$ for $2 \mathrm{~h}$ in a double planetary mixing equipment. The mixture was poured into a $200 \times 200 \mathrm{~mm}$ stainless pictureframe mold with a thickness of $2 \mathrm{~mm}$. The mold was placed in a plate vulcanizing instrument at $165^{\circ} \mathrm{C}$ curing temperature with inert gas protection. A pressure of $10 \mathrm{MPa}$ was applied and held for 5 min to obtain a group of the conductive silicone rubber sheets. The sheets were kept at room temperature $\left(20 \pm 5^{\circ} \mathrm{C}\right)$ for $24 \mathrm{~h}$ before testing. Using the method above, conductive silicone rubber samples filled with spherical, flaky and chainspherical carbonyl nickel powder were, respectively, prepared.

To effectively compare the electromagnetic properties of the rubbers with the three powders, the same volume resistivity was obtained by powder addition adjustment. Thus, the mass ratio of the spherical carbonyl nickel powder to the rubber was $3: 1$, the mass ratio of the flaky powder to the rubber was $2: 1$, and the mass ratio of the chain-spherical powder to the rubber was 1.2:1.

\section{Mechanical Test}

Tensile properties were measured according to the standard of ASTM: D412-06ae2 using a tensile testing machine (LLOYD; Lloyd Instruments, UK) at a crosshead speed of $500 \mathrm{~mm} / \mathrm{min}$.

\section{Measurements of SE}

The SE of the sample for plane-wave conditions was measured by means of the flange coaxial method. The setup consisted of a DN15115 SE tester which was connected to an Agilent 4396B RF network spectrum impedance analyzer. The scanning frequency ranged from $100 \mathrm{kHz}$ to $1.5 \mathrm{GHz}$. The thickness of the rubber layers was $2 \mathrm{~mm}$.

\section{Morphology Observation}

Scanning electron microscopy (SEM) (ZEISS EVO18; Zeiss, Germany) was used to observe the microstructure and distribution of the fillers of the composites and their tensile fracture morphology. The elemental distribution was evaluated by energy-dispersive $\mathrm{x}$-ray analysis (EDX).

\section{RESULTS AND DISCUSSION}

Figure 1 shows the microstructures of the carbonyl nickel powders with different powder morphologies. The carbonyl nickel powders with the spherical, flaky and chain-spherical morphologies can be observed in Fig. 1a-c. The average particle size of the powders was about $6 \mu \mathrm{m}, 6 \mu \mathrm{m}$, and $1.5 \mu \mathrm{m}$, respectively. The chain structure of the chain-spherical powder is comprised of many spherical particles of diameter $1-2 \mu \mathrm{m}$, while the average length of each branch of the chain structure is about $6 \mu \mathrm{m}$. In Fig. 1d-f, it can be seen that the spherical powder possesses a unique crystal surface morphology, the flaky powder has a smooth and thin-plate morphology, and the chain-spherical powder has a chain morphology comprised of many crystal particles.

Figure 2 shows the tensile fracture morphology of the conductive silicone rubber filled with the carbonyl nickel powders with different powder morphologies. The spherical, flaky and chain-spherical carbonyl nickel powder have been separately surrounded by silicone rubber and uniformly distributed. The powder particles connect with each other and form an effective conductive network, which benefits the electromagnetic shielding effectiveness (SE) of the conductive silicone rubber.

Figure 3 shows the electromagnetic $\mathrm{SE}$ of the conductive silicone rubbers filled with the spherical, flaky, and chain-spherical carbonyl nickel powders. 

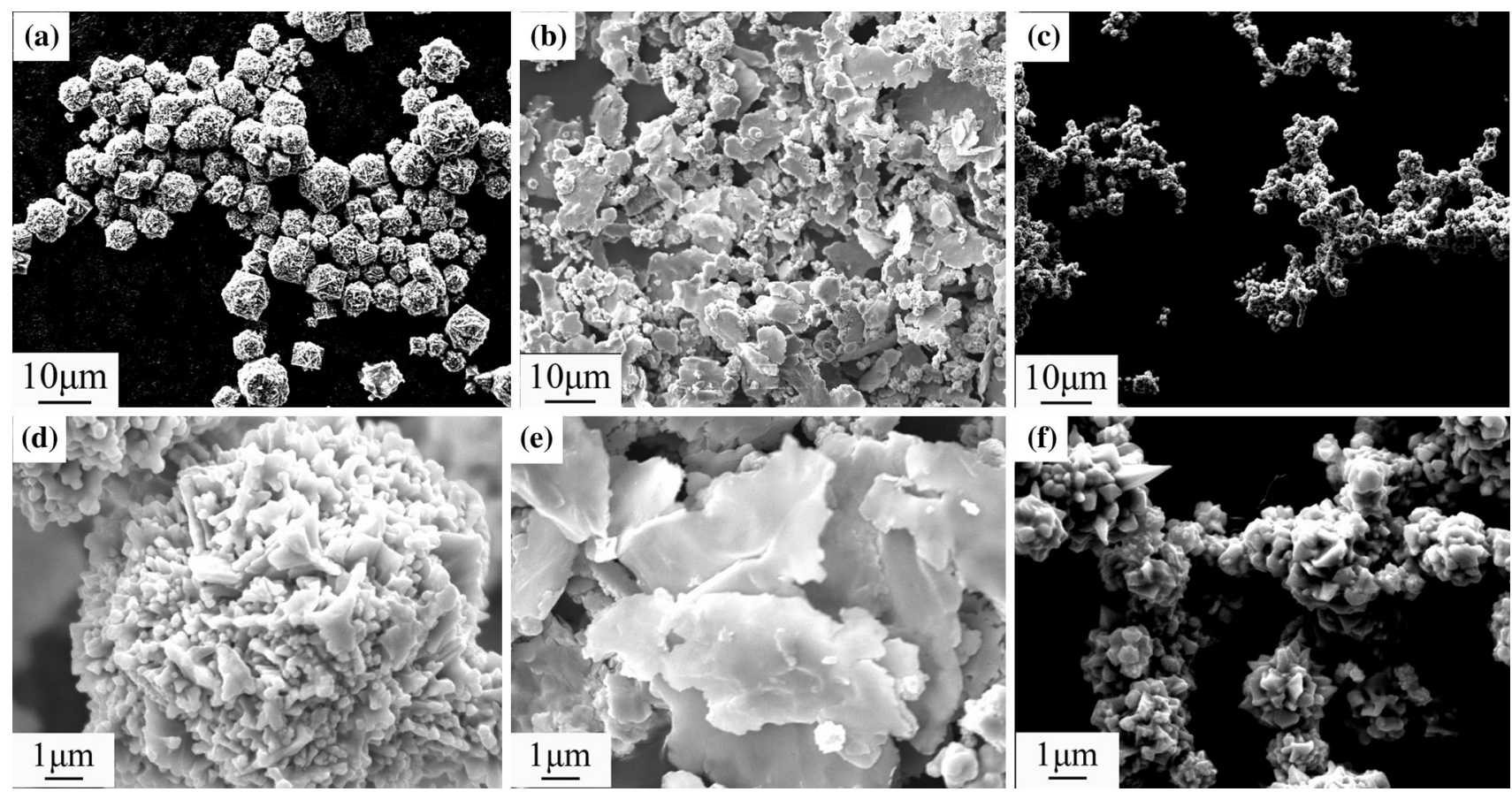

Fig. 1. Microstructures of the carbonyl nickel powders with different morphologies: (a) spherical $(\times 1 \mathrm{k})$, (b) flaky $(\times 1 \mathrm{k})$, (c) chain-spherical $(\times 1 \mathrm{k})$, (d) spherical $(\times 8 \mathrm{k})$, (e) flaky $(\times 8 \mathrm{k}),(\mathrm{f})$ chain-spherical $(\times 8 \mathrm{k})$.
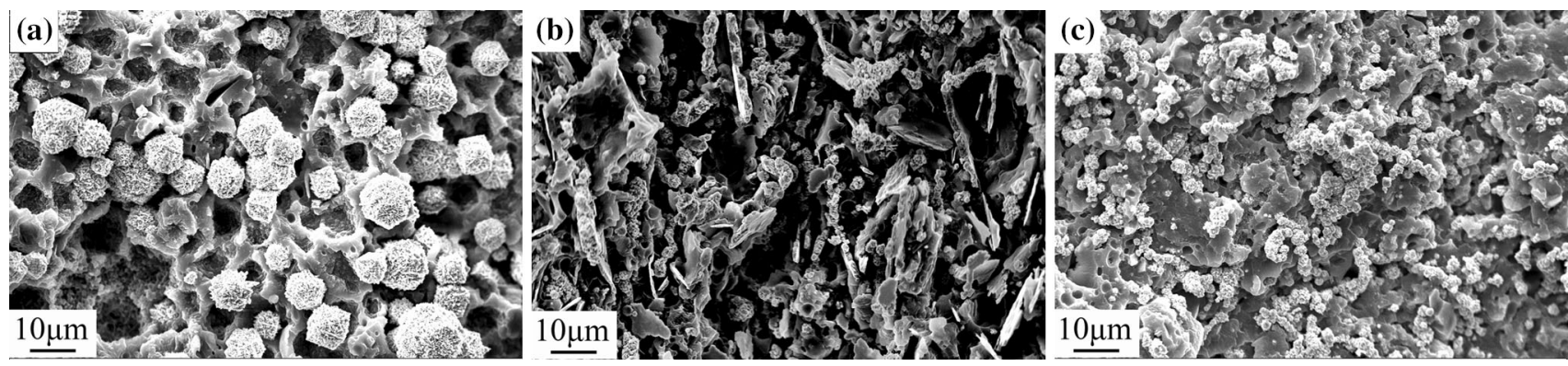

Fig. 2. Tensile fracture morphology of the conductive silicone rubber filled with (a) spherical, (b) flaky, and (c) chain-spherical carbonyl nickel powders.

At the same volume resistivity of $0.32 \Omega \mathrm{cm}$, the $\mathrm{SE}$ of the conductive silicone rubbers varies with their powder morphologies: the SE increases from the spherical andthe chain-spherical to the flaky in the frequency range of $100-400 \mathrm{MHz}$, while it rises from the spherical and the flaky to the chainspherical in the frequency range of $500-1500 \mathrm{MHz}$.

The difference in the electromagnetic $\mathrm{SE}$ is related to the carbonyl nickel powder morphologies. According to the electromagnetic shielding theory, the electromagnetic shielding property of materials is mainly due to their electromagnetic wave reflection and absorption abilities. The reflection arises from the interaction between the conducting powder particles of the material and the electromagnetic wave. The value of the reflection loss $R$ can be described as:

$$
R=\left(Z_{\mathrm{o}}-Z_{\mathrm{i}}\right) /\left(Z_{\mathrm{o}}+Z_{\mathrm{i}}\right)
$$

where $Z_{0}$ is the wave impedance of free space and its value is $377 \Omega$, and $Z_{\mathrm{i}}$ is effective impedance and its value is connected to the relative conductivity $\sigma_{\mathrm{r}}$ and the relative permeability $\mu_{\mathrm{r}}$, which is as follows:

$$
Z_{\mathrm{i}}=\sqrt{\mu_{\mathrm{r}} / \sigma_{\mathrm{r}}}
$$

Thus, it is known that the reflection loss $R$ can increase with the decrease of $\mu_{\mathrm{r}} / \sigma_{\mathrm{r}}$.

The electromagnetic wave absorption of materials is the result of the interaction between the magnetic dipole of the materials and the electromagnetic wave. During the interaction, the energy of the electromagnetic wave is converted into heat energy of which there are losses. The absorption loss $A$ can be obtained as follows: 


$$
A=131 t \sqrt{f \mu_{\mathrm{r}} \sigma_{\mathrm{r}}}
$$

in which, $t$ is the transmission distance of the electromagnetic wave in the material, and $f$ is the frequency of the electromagnetic wave. From the formula, it is detected that the absorption loss $A$ can ascend with the increase of $\mu_{\mathrm{r}} \sigma_{\mathrm{r}}$.

In the conductive silicone rubber, it is well known that the electromagnetic wave reflection and absorption abilities depend on the conductive structure of the material. As seen in Fig. 2, the different

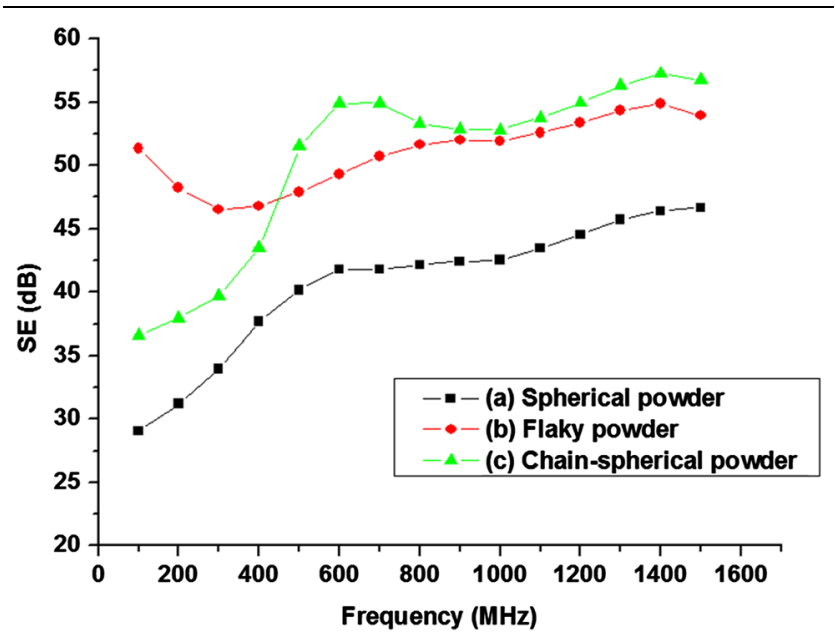

Fig. 3. Electromagnetic shielding effectiveness of the conductive silicone rubbers filled with (a) spherical, (b) flaky, and (c) chainspherical carbonyl nickel powders. carbonyl nickel powder morphology leads to the distinctive structure of the conductive network in the conductive silicone rubber. The network structures with morphologies have a different effect on the incident electromagnetic wave as shown in Fig. 4, although they result in the same volume resistivity. For the flaky and chain-spherical powders, it is observed that their particles form a more compact network structure in the rubber than the spherical ones in Fig. 2. Due to the powders' shape and structure, the flaky and chain-spherical powders have more particles to reflect and absorb the electromagnetic wave than the spherical ones in the rubber with the same volume. When the incident electromagnetic wave comes into the conductive silicone rubber, the compact network structure of the flaky and chain-spherical powders can absorb and reflect the electromagnetic wave more effectively, which reduces the penetration of the electromagnetic wave and optimizes the electromagnetic impedance of the rubber. Therefore, the reflection and absorption ability is strengthened and the SE of the rubber with the flaky and chain-spherical powders is higher than for the spherical. In addition, the flaky and chain-spherical powder particles have a different powder structure and conductive network structure in the rubber. This difference leads to the divergence of electromagnetic properties in various frequency ranges. As is known, the wavelength of the electromagnetic wave in the frequency range of $100-400 \mathrm{MHz}$ is longer than in the range of 500-1500 MHz. According to electromagnetic theory, a compact and large-area object often has a better electromagnetic shielding effect (a)

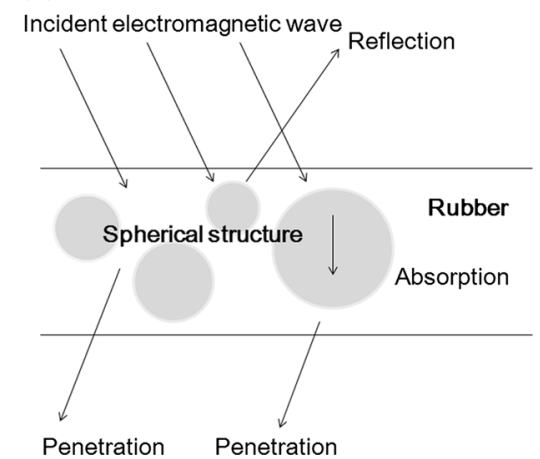

(b)

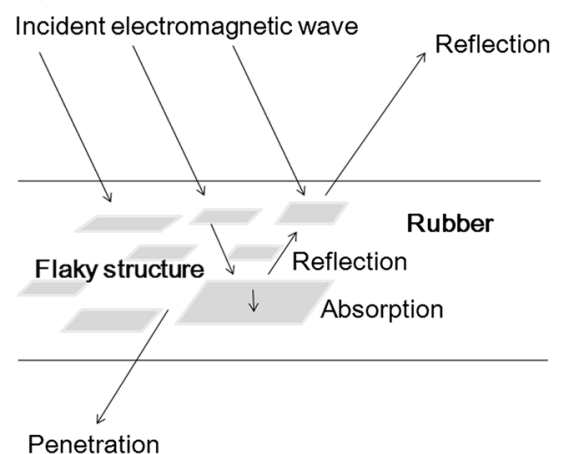

(c)

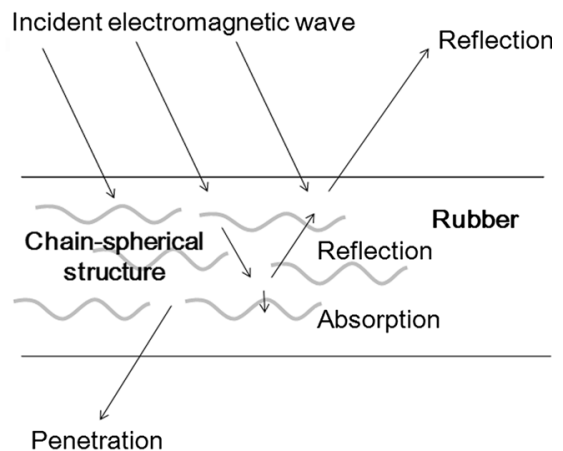

Fig. 4. Schematic diagram of the conductive network structure of the conductive silicone rubbers filled with (a) spherical, (b) flaky, and (c) chainspherical carbonyl nickel powders.

Table I. Mechanical property of the conductive silicone rubbers filled with the carbonyl nickel powders

\begin{tabular}{lcc}
\hline Powder morphology & Ultimate tensile strength (MPa) & Elongation (\%) \\
Spherical powder & 2.86 & 207 \\
Flaky powder & 3.61 & 201 \\
Chain-spherical powder & 3.85 & 185 \\
\hline
\end{tabular}


on the long-wavelength wave than the loose and small one in the same condition. Thus, the flaky powders have a relatively compact and large-area powder structure compared with the chain-spherical powders and form a multilayer plate network structure in the rubber (see Fig. 2b). The combination of the large-area and multilayer plate structure may improve the reflection ability of the electromagnetic wave in the frequency range of 100$400 \mathrm{MHz}$. As a result, the conductive network structure of the flaky powder in the rubber is more beneficial to the improvement of the $\mathrm{SE}$ in the frequency range of $100-400 \mathrm{MHz}$ than the chainspherical one.

Table I indicates the mechanical properties of the conductive silicone rubbers filled with the carbonyl nickel powders with different morphologies. As shown in Table I, the tensile strength of the rubbers increases and the elongation at break decreases from the rubbers with the spherical and flaky powder to the chain-spherical powder. This may be attributed to the morphology of the powder in the rubber. The different powder morphologies lead to the distribution of the powder particles in the silicone rubber, as shown in Fig. 2, which may cause the different deformation behavior during the tensile process.

\section{CONCLUSIONS}

In this article, the conductive silicone rubbers filled with the spherical, flaky, and chain-spherical carbonyl nickel powders have been prepared and the following conclusions are formed.

- The electromagnetic SE of the rubbers varies with their powder morphologies: the SE increases from the spherical and the chain-spherical to the flaky in the frequency range of 100 $400 \mathrm{MHz}$, while it rises from the spherical and the flaky to the chain-spherical in the frequency range of $500-1500 \mathrm{MHz}$.

- The mechanical properties of the rubbers also vary with their powder morphologies: the tensile strength of the rubbers ascends from the spherical and the flaky to the chain-spherical, while the elongation at break decreases from the sphericaland the flaky to the chain-spherical.

- The variation of the electromagnetic and mechanical propertes is attributed to the difference in the conductive network structure and the powder distribution in the rubber, which results from the interaction between the silicone rubbers and the spherical, flaky, and chain-spherical powders.

\section{ACKNOWLEDGEMENT}

The authors greatly appreciate the financial support by the State Grid Corporation of China and the Global energy Interconnection Research Institute.

\section{REFERENCES}

1. R. Wang, H. Yang, J. Wang, and G. Li, Polym. Test. 38, 53 (2014).

2. H. Zou, L.Q. Zhang, M. Tian, S.Z. Wu, and S.H. Zhao, J. Appl. Polym. Sci. 775, 2710 (2010).

3. Y. Hu, H. Zhang, F. Li, X. Cheng, and T. Chen, Polym. Test. 29,609 (2010).

4. D. Petukhov, M. Kirikova, A. Bessonov, and M. Bailey, Mater. Lett. 132, 302 (2014).

5. M. Zakaria, A. Sulong, J. Sahari, and H. Suherman, Compos. Part B Eng. 83, 75 (2015).

6. F. AL-Oqla, S. Sapuan, T. Anwer, M. Jawaid, and M. Hoque, Synth. Met. 206, 42 (2015).

7. X. Cao and H. Zhang, Appl. Surf. Sci. 264, 756 (2013).

8. X. Yin, K. Peng, A. Hu, L. Zhou, J. Chen, and Y. Du, J. Alloys Compd. 479, 372 (2009).

9. Z. Song, J. Xie, I. Zhou, X. Wang, T. Liu, and L. Deng, J. Alloys Compd. 551, 677 (2013).

10. L. Liu, Y. Duan, S. Liu, L. Chen, and J. Guo, J. Magn. Magn. Mater. 322, 1736 (2010).

11. H. Wang, D. Zhu, W. Zhou, and F. Luo, J. Magn. Magn. Mater. 375, 111 (2015)

12. Y. Duan, G. Wu, S. Gu, S. Li, and G. Ma, Appl. Surf. Sci. 258, 5746 (2012).

13. C. Yin, J. Pan, L. Bai, P. Ding, and P. Yuan, J. Magn. Magn. Mater. 340, 65 (2013).

14. X. Tian, X. Liu, H. Feng, and J. Xu, J. Alloys Compd. 484, $882(2009)$.

15. X. Li, G. Ji, H. Lv, M. Wang, and Y. Du, J. Magn. Magn. Mater. 355, 65 (2014).

16. Y. Wan, J. Xiao, C. Li, G. Xiong, R. Guo, L. Li, M. Han, and H. Luo, J. Magn. Magn. Mater. 399, 252 (2016).

17. M. Jazirehpour and S. Ebrahimi, Ceram. Int. 42, 16512 (2016). 